\title{
Sero-Prevalence of Syphilis and HIV and Associated Factors in Pregnant Women Attending Antenatal Care Clinics in Debre Berhan Public Health Institutions, Ethiopia
}

\author{
Seblewongale Zinabie ${ }^{1}$, Emishaw Belachew ${ }^{1}$, Teferi Yidenek ${ }^{2}$, Moges Lewetegn ${ }^{3}$, Tsegahun Asfaw ${ }^{3,}$ * \\ ${ }^{1}$ Department of Medicine, Debre Berhan University, Debre Berhan, Ethiopia \\ ${ }^{2}$ Field Epidemiology, North Shewa Zone Health Bureau, Debre Berhan, Ethiopia \\ ${ }^{3}$ Department of Medical Laboratory Science, Debre Berhan University, Debre Berhan, Ethiopia
}

Email address:

zinabieseblewongel@gmail.com (S.Zinabie), emishawb2004@gmail.com (E. Belachew), teferiyidenek@gmail.com (T. Yidenek), mogesil@gmail.com (M. Lewotegn), tsegahun.asfaw12@gmail.com (T. Asfaw)

${ }^{*}$ Corresponding author

\section{To cite this article:}

Seblewongale Zinabie, Emishaw Belachew, Teferi Yidenek, Moges Lewetegn, Tsegahun Asfaw. Sero-Prevalence of Syphilis and HIV and Associated Factors in Pregnant Women Attending Antenatal Care Clinics in Debre Berhan Public Health Institutions, Ethiopia. American Journal of Biomedical and Life Sciences. Vol. 6, No. 3, 2018, pp. 56-62. doi: 10.11648/j.ajbls.20180603.14

Received: June 12, 2018; Accepted: June 27, 2018; Published: July 24, 2018

\begin{abstract}
Syphilis is one of the sexually transmitted infections caused by bacteria known as Treponema pallidum and it is transmitted through sexual contact, via blood transfusion, or transplacentally from a pregnant woman to her fetus. Among STIs, syphilis and HIV are significant public health problems in Ethiopia and causes several adverse pregnancy outcomes. Thus this study aims to assess magnitude of both infections among pregnant women attending antenatal care. Facility based retrospective cross-sectional study was conducted in Debre Berhan public health institutions and data was collected using pretested checklist. Finally data was entered to EPI Data3.02 and analyzed using SPSS version21. P-value $<0.05$ was considered statistically significant difference. Among the total participants, 179 (47\%) were in the age group of 25-34 years, $220(57 \%)$ were living in urban settings and majority $256(67 \%)$ of the pregnant mothers were married. The overall prevalence of syphilis and HIV in pregnant mothers were $7(1.8 \%)$ and 28 (7.2\%) respectively. The highest number of syphilis and HIV prevalence were reported in 2015. Previous history of STD for syphilis (AOR 9.4; 95\% CI 1.6-25) and HIV (AOR 8.2; 95\% CI 2.2-31.8), previous history of still birth for syphilis (AOR 4.9; 95\% CI 1.1-23.4) and HIV (AOR 5.6; 95\% CI 1.8-17.1) and partner HIV test positive for HIV (AOR 11.9; 95\%CI 1.18-20.6) are risk factor. The result shows syphilis and HIV infections were prevalent among pregnant women, indicating that they are still significant public health problems. Therefore, there should be health education for every pregnant woman regarding prevention of the two infection and partner testing is recommended.
\end{abstract}

Keywords: Associated Factors, HIV, Pregnant Mather, Sero-prevalence, Syphilis

\section{Introduction}

Sexually transmitted infections (STIs) are an emerging public health concern, especially in developing countries ([13]). Syphilis, a genital ulcerative disease caused by the bacterium Treponema pallidum, is associated with significant complications if left untreated and can facilitate the transmission and acquisition of HIV infection ([4-6]). Both Syphilis and HIV are transmitted through sexual contact, with infectious lesions of the mucous membranes or abraded skin, via blood transfusion, or in utero by transplacental passage from a pregnant woman to her fetus $([7,8])$. Globally, an estimated 1.3 billion people are suffering from STIs $[9,10]$. In Africa, a significant portion of pregnant women have been suffering from STIs, and it is the major cause of maternal and perinatal morbidity ([9-11]).

HIV and syphilis infections are epidemiologically interrelated, and co infection is common. The biological explanation for enhanced susceptibility to HIV among individuals infected with syphilis is based on the theory that the break down in mucosal integrity due to ulceration 
provides a portal of entry for the HIV virus. In addition, this ulceration may result in an influx of CD4 lymphocytes locally increasing the number of HIV target cells [7].

In Ethiopia, little is known about what predisposes pregnant women to syphilis and HIV infection. Therefore, this study aims to study trends and sero-prevalence of Syphilis and HIV infections and associated factors among pregnant women.

\section{Methodology}

\subsection{Study Area and Periods}

The study was conducted in Debrebirhan, North Shewa Zone of Amhara Region about 120 kilometers from north east of Addis Ababa. The town has 1 Hospital, 3 health centers and 14 health posts which are currently on service.

\subsection{Study Design and Study Period}

Facility based retrospective cross-sectional study was conducted to assess sero-prevalence of syphilis and HIV infections and associated factors among pregnant women attending antenatal care in public health institutions at Debre Berhan town from September 2015 to August 2017

\subsection{Sample Size Determination}

Sample size was statistically calculated based on single population proportion formula by taking $9.0 \%$ prevalence of syphilis infection from previous study [12] and desired precision of $3 \%$. A $95 \%$ confidence level and $10 \%$ contingency was considered. Finally, a total of 385 study participant was included.

\subsection{Sampling Technique and Procedure}

One hospital and three health centers were selected in the town that gives antenatal care services. A total of 10,308 pregnant women were registered at the antenatal care clinics at all public health institutions in the town since Sep 2015 and August 2017. Samples proportional to pregnant women in the selected health facilities was allocate. Lists of pregnant women were prepared using unique identification numbers from records found in ANC clinics and sampled by systematic random sampling using the registers sampling frame. To select the first eligible study participant, a simple random sampling method was used and the study units was selected until the required sample size were achieved. All pregnant women who had ANC records with complete information was included in the study while all pregnant women who had ANC records with incomplete information were excluded.

\subsection{Data Collection Method}

Data was collected by trained nurses from record log book and patient charts using structured checklist. The checklist was pre-tested on the ANC chart on randomly selected non included year and another health center which provides ANC service and which is not part of the study and necessary adjustments were made in the data collection instrument.

\subsection{Data Entry and Analysis Plan}

The data was entered by using EpiData3.02 and was analyzed using SPSS version 21. P-value less than 0.05 were taken as significant association.

\subsection{Ethical Consideration}

An ethical approval was obtained from Debre Berhan University ethical review and research committee. Permission was obtained from administration of Debre Berhan public health institutions, where the study was carried out

\section{Result}

\subsection{Socio-demographic Characteristics}

Among the total participants, 179 (47\%) were in the age group of 25-34 years, $220(57 \%)$ were living in urban settings and majority $256(67 \%)$ of the pregnant mothers were married (Table 1).

Table 1. Socio-demographic characteristics of pregnant women attending at Debre Berhan public health institutions, Debre Berhan, Ethiopia, 2018.

\begin{tabular}{ll}
\hline Variables & Frequency N (\%) \\
\hline Age & $117(30.5)$ \\
$15-24$ & $179(46.5)$ \\
$25-34$ & $89(23.0)$ \\
$35-49$ & \\
Residence of the mother & $220(57.0)$ \\
Urban & $165(43.0$ \\
Rural & \\
Mother occupation & $83(21.6)$ \\
Farmer & $114(29.6)$ \\
House wife & $44(11.4)$ \\
Student & $49(12.7)$ \\
Daily laborer & $49(12.7)$ \\
Merchant & $46(12.0)$ \\
Gov't employed & \\
Marital status & $256(66.5)$ \\
Married & $68(17.6)$ \\
Single & $37(9.7)$ \\
Divorced & $18(4.7)$ \\
Widowed & $6(1.5)$ \\
Note recorded &
\end{tabular}

\subsection{Prevalence of HIV and Syphilis Infection}

A total of 385 pregnant women were enrolled in the study. Overall, $7(1.8 \%)$ and $28(7.2 \%)$ of the pregnant women were positive for syphilis and HIV respectively. One (2.8\%) of pregnant women had both syphilis/HIV co-infections (Table 2). 
Table 2. Socio-demographic, Sexual behavior and Obstetric risk factors related to syphilis infection in pregnant women attending ANC at Debre Berhan public health institutions, Debre Berhan, Ethiopia,2018.

\begin{tabular}{|c|c|c|c|c|}
\hline Variables & Categories & Reactive N (\%) & Non-reactive N (\%) & COR $(95 \%$ Cl), P-value \\
\hline \multirow{3}{*}{ Age } & $15-24$ & $1(14.3)$ & $116(30.6)$ & $2.6(0.23-9.2), \mathrm{p}=0.42$ \\
\hline & $25-34$ & $4(57.1)$ & $175(46.4)$ & $1.0(0.18-58.2), \mathrm{p}=0.99$ \\
\hline & $35-49$ & $2(28.6)$ & $87(23.0)$ & 1 \\
\hline \multirow{2}{*}{ Residency } & Urban & $2(28.6)$ & $218(57.6)$ & $1.8(0.90-6.2), \mathrm{p}=0.43$ \\
\hline & Rural & $5(62.4)$ & $160(42.4)$ & 1 \\
\hline \multirow{5}{*}{ Marital status } & Married & $3(42.8)$ & $253(67.0)$ & $0.40(.06-2.3), \mathrm{p}=0.31$ \\
\hline & Single & $2(28.6)$ & $66(17.5)$ & $0.42(0.04-2.2), \mathrm{p}=0.46$ \\
\hline & Divorced & $1(14.3)$ & $36(9.5)$ & $0.20(0.02-2.0), \mathrm{p}=0.17$ \\
\hline & Widowed & $1(14.3)$ & $17(4.5)$ & 1 \\
\hline & Not recorded & 0 & $6(1.5)$ & \\
\hline \multirow{6}{*}{ Occupation } & Farmer & $1(14.3)$ & $82(21.7)$ & $1.8(0.11-9.8), p=0.67$ \\
\hline & Housewife & $2(28.5)$ & $112(29.6)$ & $1.2(0.11-7.2), p=0.86$ \\
\hline & Student & $1(14.3)$ & $43(11.3)$ & $.90(0.05-5.7), p=0.97$ \\
\hline & Daily laborer & $1(14.3)$ & $48(12.7)$ & $1.1(0.06-7.5), p=0.96$ \\
\hline & Merchant & $1(14.3)$ & $48(12.7)$ & $1.1(0.06-7.5), p=0.96$ \\
\hline & Employee & $1(14.3)$ & $45(12.0)$ & 1 \\
\hline \multirow{2}{*}{ Mother history of STD } & Yes & $2(28.6)$ & $15(4.0)$ & $9.6(1.7-21.2), p=0.010$ \\
\hline & No & $5(71.4)$ & $363(96.0)$ & 1 \\
\hline \multirow{2}{*}{ Gravidity } & Primi gravidae & $2(28.6)$ & $120(31.7)$ & $1.2(0.22-6.1), p=0.85$ \\
\hline & Multi gravidae & $5(71.4)$ & $258(68.3)$ & 1 \\
\hline \multirow{4}{*}{ Pregnancy status } & First & $1(14.3)$ & $113(30.0)$ & 1 \\
\hline & Second & $3(42.8)$ & $131(34.5)$ & $1.6(0.26-9.9), p=0.60$ \\
\hline & Third & $1(14.3)$ & $80(21.2)$ & $2.9(0,26-13.4), p=0.38$ \\
\hline & Fourth\& above & $2(28.6)$ & $54(14.3)$ & $4.2(0.37-27.2), p=0.25$ \\
\hline \multirow{2}{*}{ History of still birth } & Yes & $3(42.8)$ & $49(13.0)$ & $5.0(1.1-23.1), \mathrm{p}=0.038$ \\
\hline & No & $4(57.2)$ & $329(87.0)$ & 1 \\
\hline \multirow{2}{*}{ Transfusion } & Yes & $1(14.3)$ & $8(21.2)$ & $7.7(0.83-17.6), p=0.073$ \\
\hline & No & $6(85.7)$ & $370(97.8)$ & 1 \\
\hline \multirow{2}{*}{ History of abortion } & Yes & $1(14.3)$ & $16(4.2)$ & $3.8(0.43-13.2), \mathrm{p}=0.20$ \\
\hline & No & $6(85.7)$ & $362(95.8)$ & 1 \\
\hline \multirow{2}{*}{ History of LBW } & Yes & $1(14.3)$ & $7(2.0)$ & $8.8(4.92-13.4), \mathrm{p}=0.057$ \\
\hline & No & $6(85.7)$ & $371(98.0)$ & 1 \\
\hline \multirow{4}{*}{ Number of delivery } & One & $3(42.8)$ & $133(35.2)$ & $1.8(0.19-18.0), \mathrm{p}=0.59$ \\
\hline & Two & $1(14.3)$ & $82(21.7)$ & $0.47(0.07-2.9), \mathrm{p}=0.42$ \\
\hline & Greater $>=$ three & $2(28.5)$ & $42(11.0)$ & \\
\hline & Zero & $1(14.3)$ & $121(32.0)$ & \\
\hline \multirow{2}{*}{ HIV status } & Reactive & $2(28.6)$ & $26(7.0)$ & $5.4(1.1-19.2), \mathrm{p}=0.05$ \\
\hline & Non-reactive & $5(71.4)$ & $352(93.0)$ & 1 \\
\hline
\end{tabular}

\subsection{Trend of Syphilis and HIV}

Among pregnant women in Debre Berhan public health institutions, the prevalence of syphilis and HIV between $2015 / 16$ and $2016 / 17$ were $1.8 \%$ and $7,2 \%$ respectively. The highest number of syphilis and HIV prevalence were reported in $2015 / 16$ (Figure 1).

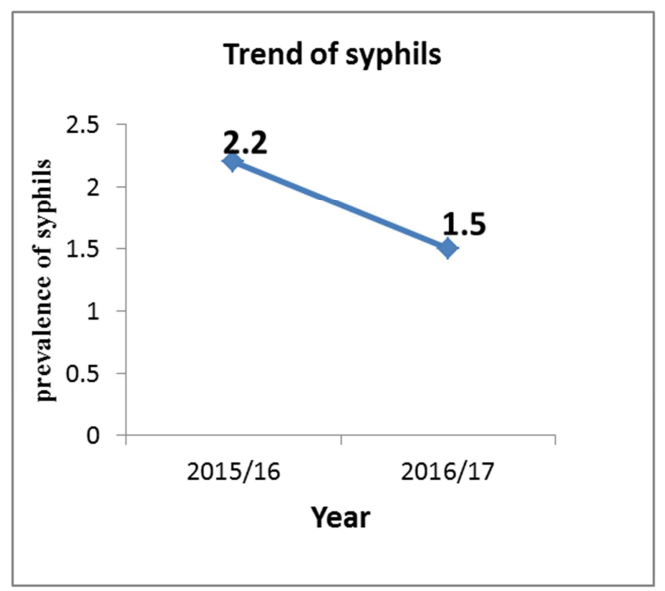

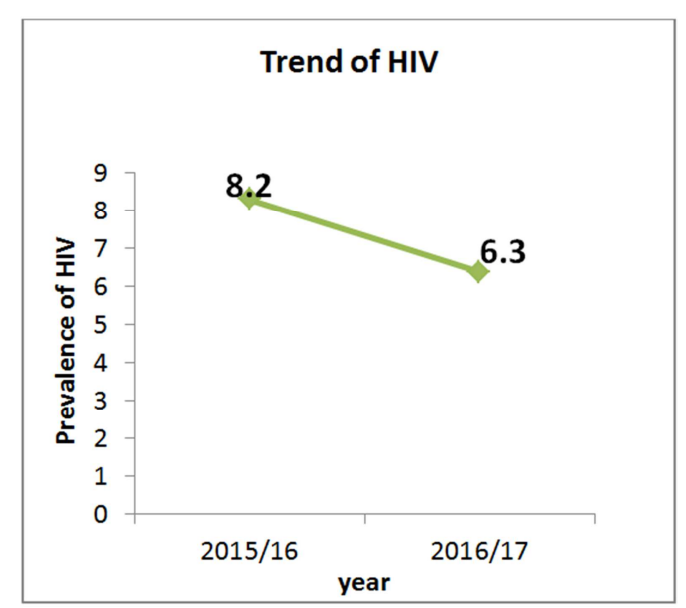

Figure 1. Syphilis and HIV prevalence by year from 2015/16-2016/17 in Debre Berhan public health institutions, Debre Berhan, Ethiopia, 2018.

\subsection{Socio-demographic, Sexual Behavior and Obstetric Risk Factors for Acquiring Syphilis}

About $57 \%$ of the syphils sero-positive mothers were 
found in 25-34 age categories. The prevalence of syphilis was $3.0 \%$ and $0.9 \%$ in rural and urban respectively. Similarly of total reactive for VDRL test, more than $71 \%$ of syphilis sero-positive was from rural area. The prevalence of syphilis was higher among widowed (5.6\%). With regard to occupation of the mothers, the prevalence of syphilis was higher among those students $(2.3 \%)$.

Sexual behavior related risk factor for syphilis in bivariate analysis was: mothers having previous history of STD (COR; 9.6 95\%CI 1.7-21.2, $\mathrm{p}=0.010$ ), history of still birth (COR; $5.095 \% \mathrm{CI}$ 1.1-23.1, $\mathrm{p}=0.038$ ) and HIV status (COR; 5.4(95\%CI 1.1-19.2, $\mathrm{p}=0.05)$. However, age, residency, marital status, occupation, gravidity, pregnancy status, number of delivery, history of blood transfusion and history of abortion were not a determinant for syphilis (Table 2).

\subsection{Socio-demographic, Sexual Behavior and Obstetric Risk Factors for Acquiring HIV}

About $85.7 \%$ of the HIV sero-positive mothers were found in 25-49 age categories. The prevalence of HIV was $6.1 \%$ and $8.2 \%$ in rural and urban respectively. The prevalence of HIV was $4.4 \%$ among single and $8.2 \%$ among married women. With regard to occupation of the mothers the prevalence of HIV was high among that government employee (13\%).

Sexual behavior and obstetric risk factors for acquiring HIV in bivariate analysis were: age 15-24 (COR; 4.4 95\%CI 1.4-14.0, $\mathrm{p}=0.013)$, mothers having previous history of STD (COR; $6.295 \%$ CI 2.0-19.2, $\mathrm{p}=0.001$ ), history of still birth (COR; 4.2 95\%CI 1.8-9.6, $\mathrm{p}=0.001$ ), gravidity (COR; 4.2 $95 \%$ CI $1.2-14.1, \mathrm{p}=0.02)$, number of delivery of $>=3(\mathrm{COR}$; $0.1995 \%$ CI $0.06-0.60, \mathrm{p}=0.006$ ), syphilis status (COR; 5.4 95\% CI 1.1-19.2, $\mathrm{p}=0.05$ ) and partner HIV status (COR;28 $95 \%$ CI 3.5-220, $\mathrm{p}=0.002$. However, residency, marital status, occupation, pregnancy status, blood transfusion, history of abortion, pregnancy status and history of LBW were not a determinant for HIV (Table 3).

Table 3. Socio-demographic, Sexual behavior and Obstetric risk factors related to HIV infection in pregnant women attending ANC at Debre Berhan public health institutions, Debre Berhan, Ethiopia, 2018.

\begin{tabular}{|c|c|c|c|c|}
\hline Variables & Categories & Reactive N (\%) & Non-reactive N (\%) & COR (95\%CI). P-value \\
\hline \multirow{3}{*}{ Age } & $15-24$ & $4(14.3)$ & $113(31.7)$ & $4.4(1.4-14.0), \mathrm{p}=0.013$ \\
\hline & $25-34$ & $12((42.9)$ & $167(46.8)$ & $2.1(0.93-5.0), \mathrm{p}=0.072$ \\
\hline & $35-49$ & $12(42.8)$ & $77(21.5)$ & 1 \\
\hline \multirow{2}{*}{ Residency } & Urban & $18(64.3)$ & $202(56.6)$ & $1.4(0.60-3.01), p=0.43$ \\
\hline & Rural & $10(35.7)$ & $155(43.4)$ & 1 \\
\hline \multirow{5}{*}{ Marital status } & Married & $21(75.0)$ & $235(65.8)$ & 1 \\
\hline & Single & $3(10.8)$ & $65(18.2)$ & $0.70(0.15-3.3), p=0.67$ \\
\hline & Divorced & $2(7.1)$ & $35(9.8)$ & $1.5(0.35-6.9), \mathrm{p}=0.55$ \\
\hline & Widowed & $2(7.1)$ & $16(4.5)$ & $1.9(0.56-6.6), p=0.29$ \\
\hline & Not recorded & & $6(1.7)$ & \\
\hline \multirow{6}{*}{ Occupation } & Farmer & $4(14.4)$ & $79(22.1)$ & $2.9(0.8-11), p=0.11$ \\
\hline & Housewife & $7(25.0)$ & $107(30.0)$ & $2.3(0.72-7.2), p=0.15$ \\
\hline & Student & $3(10.7)$ & $41(11.5)$ & $2.0(0.48-8.7), p=0.33$ \\
\hline & Daily laborer & $5(17.8)$ & $44(12.3)$ & $1.3(0.38-4.7), p=0.64$ \\
\hline & Merchant & $3(10.7)$ & $46(12.9)$ & $2.3(0.52-9.5), p=0.27$ \\
\hline & Employee & $6(21.4)$ & $40(11.2)$ & 1 \\
\hline \multirow{2}{*}{ Mother history of STD } & Yes & $5(17.8)$ & $12(3.4)$ & $6.2(2.0-19.2), p=0.001$ \\
\hline & No & $23(82.2)$ & $345(96.6)$ & 1 \\
\hline \multirow{2}{*}{$\begin{array}{l}\text { History of blood } \\
\text { transfusion }\end{array}$} & Yes & $2(7.2)$ & $7(2.0)$ & $3.8(0.76-19.4), p=0.103$ \\
\hline & No & $26(92.8)$ & $350(98.0)$ & 1 \\
\hline \multirow{2}{*}{ Gravidity } & Primi gravida & $3(10.7)$ & $119(33.3)$ & 1 \\
\hline & Multi gravida & $25(89.3)$ & $238(66.7)$ & $4.2(1.2-14.1), p=0.022$ \\
\hline \multirow{4}{*}{ Pregnancy status } & First & $4(14.4)$ & $110(30.8)$ & 1 \\
\hline & Second & $8(28.5)$ & $126(35.3)$ & $1.9(0.60-5.7), p=0.26$ \\
\hline & Third & $10(35.7)$ & $71(18.9)$ & $0.85(0.30-2.4), p=0.77$ \\
\hline & Fourth and above & $6(21.4)$ & $50(14.0)$ & $3.3(0.91-12.2), p=0.074$ \\
\hline \multirow{4}{*}{ Number of delivery } & One & $6(21.4)$ & $130(36.3)$ & $0.93(0.27-3.1), p=0.90$ \\
\hline & Two & $9(32.1)$ & $74(20.2)$ & $0.35(0.11-1.1), p=0.70$ \\
\hline & Greater $>=3$ & $8(28.6)$ & $36(10.1)$ & $0.19(0.06-0.60), p=0.006$ \\
\hline & Zero & $5(17.9)$ & $117(32.6)$ & 1 \\
\hline \multirow{2}{*}{$\begin{array}{l}\text { Previous still birth or } \\
\text { neonatal loss }\end{array}$} & Yes & $10(35.7)$ & $42(11.8)$ & $4.2(1.8-9.6), p=0.001$ \\
\hline & No & $18(64.3)$ & $315(88.2)$ & 1 \\
\hline \multirow{2}{*}{ History of abortion } & Yes & $1(3.6)$ & $16(4.5)$ & $1.3(0.16-9.98 .7), p=0.88$ \\
\hline & No & $27(96.4)$ & $341(95.5)$ & 1 \\
\hline \multirow{2}{*}{ History of LBW } & Yes & $1(3.6)$ & $7(2.0)$ & $1.9(0.22-15.6), p=0.57$ \\
\hline & No & $27(96.4)$ & $350(98.0)$ & 1 \\
\hline \multirow{2}{*}{ Syphilis status } & Reactive & $2(7.1)$ & $5(1.4)$ & $5.4(1.1-19.2), p=0.05$ \\
\hline & Non-reactive & $26(92.9)$ & $352(98.6)$ & 1 \\
\hline \multirow{3}{*}{ Partner HIV test } & Positive & $4(14)$ & $2(1.0)$ & $28(3.5-220), p=0.002$ \\
\hline & Negative & $3(11)$ & $42(12.0)$ & 1 \\
\hline & Not recorded & $21(75)$ & $313(87.0)$ & \\
\hline
\end{tabular}


Multivariable logistic regression analysis

Variables with a P-value $<0.25$ in bivariate analysis were again entered in to multivariate analysis phase to check out the presence of confounders, if any. On Multivariate analyses the independent risk factors for syphilis (Mother history of STD and history of still birth) and for HIV (Mother history of STD, history of still birth\& partner HIV status).

Mothers who had previous history of STD were 9.4and 8.2 times to acquire syphilis (AOR 9.4; 95\% CI 1.6-25) and HIV (AOR 8.2; 95\% CI 2.2-31.8) respectively. Those mothers who had previous history still birth were 4.9 and 5.6 to develop syphilis (AOR 4.9; 95\% CI 1.1-23.4) and HIV (AOR 5.6; 95\% CI 1.8-17.1) respectively. Those pregnant mothers whose partner HIV test positive were 11.9 times to acquire HIV (AOR 11.9; 95\%CI 1.18-20.6) (Table 4).

Table 4. Factors independently associated with Syphilis and HIV infection in pregnant women attending ANC at Debre Berhan public health institutions, Debre Berhan, Ethiopia, 2018.

\begin{tabular}{|c|c|c|c|c|c|}
\hline Variables Categories & & Reactive N (\%) & Non-reactive N (\%) & COR & AOR, 95\% CI, P-value \\
\hline \multicolumn{6}{|c|}{ Factors independently associated with Syphilis } \\
\hline Mother history of STD & $\begin{array}{l}\text { Yes } \\
\text { No }\end{array}$ & $\begin{array}{l}2(28.6) \\
5(71.4)\end{array}$ & $\begin{array}{l}15(4.0) \\
363(96.0)\end{array}$ & $9.6(1.7-21.2)$ & $9.4(1.6-25), p=0.013$ \\
\hline History of still birth & $\begin{array}{l}\text { Yes } \\
\text { No }\end{array}$ & $\begin{array}{l}3(42.9) \\
4(57.1)\end{array}$ & $\begin{array}{l}49(13.0) \\
329(87.0)\end{array}$ & $5.0(1.1-23.1)$ & $4.9(1.1-23.4), p=0.046$ \\
\hline \multicolumn{6}{|c|}{ Factors independently associated with HIV } \\
\hline Mother history of STD & $\begin{array}{l}\text { Yes } \\
\text { No }\end{array}$ & $\begin{array}{l}5(17.8) \\
23(82.2)\end{array}$ & $\begin{array}{l}12(3.4) \\
345(96.6)\end{array}$ & $6.2(2.0-19.2)$ & $8.2(2.2-31.8), \mathrm{p}=0.002$ \\
\hline History of still birth & $\begin{array}{l}\text { Yes } \\
\text { No }\end{array}$ & $\begin{array}{l}10(35.7) \\
18(64.3)\end{array}$ & $\begin{array}{l}42(11.8) \\
315(88.2)\end{array}$ & $4.2(1.8-9.6)$ & $5.6(1.8-17.1), p=0.003$ \\
\hline Partner HIV test & $\begin{array}{l}\text { Positive } \\
\text { Negative } \\
\text { Not recorded }\end{array}$ & $\begin{array}{l}4(14.0) \\
3(11.0) \\
21(75) \\
\end{array}$ & $\begin{array}{l}2(1.0) \\
42(12 .) \\
313(87.0)\end{array}$ & $28(3.5-220)$ & $11.9(1.18-20.6), \mathrm{p}<0.036$ \\
\hline
\end{tabular}

\section{Discussion}

The prevalence of syphilis in this study $(1.8 \%)$ was lower than the study done at Gondar (3.7\%) [13], Gondar teaching Hospital (2.9\%) [14], Addis Ababa (2.9\%) [15]. However, roughly similar with national ANC sentinel survey of Ethiopia, in $2014(1.2 \%)$ [16] and the study done at wolemera district (1.2\%) [17]. However, it was higher than previous studies conducted in Northwest Ethiopia (1\%) [18]. This may be due to the difference in sexual behavior and practices, climatic conditions, type of population being studied, and socio demographic characteristics of the study populations.

The prevalence of syphilis in this study was higher in rural $(3.1 \%)$ than urban $(0.9 \%)$ which goes in line with the national ANC sentinel survey of Ethiopia in 2009 (6.7\%) in rural and (4.7\%) from urban setting [19]. Similarly, national ANC sentinel survey of Ethiopia, in 2014 (0.7\%) in urban; likewise the syphilis prevalence in 2012 round were $0.7 \%$ in urban and $1 \%$ in rural sites [17]. But in contrary to study conducted at Wolmera district, $(0.3 \%)$ in rural and $(1.9 \%)$ in urban [18], this shows that in majority of the study, prevalence of syphilis increased in rural than urban. It could be due lack of awareness, poor knowledge, poor understanding of health service at the ANC in rural pregnant women which help for adequate prevention of sexually transmitted diseases. Urban residents might have better health seeking behavior to get tested and treated earlier which could alter the status of syphilis.

In this study, the prevalence of syphilis were higher among the pregnant women with the age of 25-34 and 35-49. This finding was consistent with finding at Gondar [14], Wolmera district [17] and 2014 ANC survey done in Ethiopia [16]. Prevalence of syphilis in this study are relatively higher in widowed by marital status $(5.6 \%)$ and student in occupation $(2.3 \%)$. The possible explanation for this finding could be that, they may have higher chance of multiple sexual partners, which may increase the chance of the infection.

Pregnant women who had previous history of STD had 9 times [AOR $(95 \% \mathrm{CI})$ 9.4(1.6-25); $\mathrm{p}=0.013$ ] significantly increased chance of syphilis infection compared to their counterpart. This result also shows that the prevalence of syphilis is 5 times increased with history of still birth [AOR (95\% CI) 4.9(1.1-23.4), $\mathrm{p}=0.046]$.

In this study prevalence of HIV among pregnant women (7.2\%) was lower than a study done at Gondar (10.33\%) [13], Gondar teaching hospital $(9.6 \%)$ [14]. In contrast to this, the result in this study shows relatively higher prevalence of HIV as compared to the study done at eastern Africa (4.4\%) [21], the national unadjusted HIV prevalence (3.0\%), (urban 5.3\% and rural (1.9\%) [19], wolemera district $(2.9 \%)$ [17]. The prevalence of HIV in this study was higher in urban $(8.2 \%)$ than in rural $(6.1 \%)$, this result is consistence with the national surveillance result 2014 [16] and study conducted at Wolmera district [17]. This may be due females living in urban area are more likely to engaged in early sexual practice \& that having multiple sexual partners. Activities like population movement, harmful sexual behavior; long distance driver and commercial sex worker are more widespread in urban areas. Majorities of the present study population were from urban resident and this may be attributed for higher urban HIV prevalence.

Majority of HIV positive participants in this study were among the age group 25 to $34,13.6 \%$, which goes in line with study done at wolemera district [17], national surveillance done 
in 2014 [16]. Women in this age group are highly vulnerable to HIV infection because this age group comprises a sexually active segment of the population. Higher prevalence of HIV in our study was among married women, similar with study done at Gondar [13]. The higher number of the respondent in the study was married that showed the highest prevalence it might be as a fact of numerical advantage among married women (high proportion of married women in the study). Moreover they are less likely to use protective methods like condom and mostly uncommon among married couples and married women also have more chance for repeated sexual contact, as a result they may has higher prevalence of HIV than others. Those pregnant mothers whose Partner HIV test positive were twelve times more risky to acquire HIV [AOR (95\%CI) 11.9; 1.18-20.6), $\mathrm{p}<0.036$ ] than those women's having HIV negative sexual partner. Similarly, mothers who had previous history of STD were eight times more risky to acquire HIV (AOR 8.2; 95\% $\mathrm{CI}$;2.2-31.8), than those pregnant women's had no history of STD and those mothers with previous history still birth also had six times higher chance to develop HIV [AOR (95\% CI) 5.6;1.817.1)], as compared to their counterpart.

The result of this study shows that one $(2.8 \%)$ of the pregnant woman had both syphilis/HIV co-infections, relatively lower than that of national (4.3\%) [22], in contrast it was higher than that of the study done at Gondar [13]. This is due to occurrence of syphilis favors the transmission of HIV to the pregnant mother. Enhanced susceptibility to HIV among individuals infected with syphilis is based on the theory that the break down in mucosal integrity due to ulceration provides a portal of entry for the HIV virus [23].

In this study, the prevalence of syphilis and HIV were decline from 2015 to $2017,2.2 \%$ to $1.5 \%$ and $8.2 \%$ to $6.3 \%$ respectively. The reason behind why the prevalence were decreased from 2015 to 2017 in this study may be as a result of delivering integrated services for pregnant women through early screening of syphilis and effective treatment of symptomatic individuals in antenatal care.

\section{Conclusion}

This study showed syphilis and HIV were prevalent among pregnant women at Debre Berhan Public Health Institutions, even though there is a decreased trends from 2015 to 2017. Prevalence of HIV is higher among pregnant women's whose age categories above 25 years and Syphilis prevalence was more strikes in those mothers from rural setup. Mother history of STD and history of still birth are the significant risk factors for both Syphilis and HIV. In general both Syphilis and HIV; still remains significant public health problems in our study area. Therefore, there should be health education about syphilis and HIV to raise the awareness of mothers.

\section{Conflict of Interest}

The authors declare that they have no conflict of interests.

\section{Authors' Contributions}

SZ and EB - Collect the data. TY- analyzed the data. TA Analyze the data and wrote the manuscript. SZ, EB, TY and ML reviewed the manuscript. TA and ML participated in its design. All authors read and approved the final manuscript.

\section{Acknowledgements}

We would like to thank Debre Berhan University for giving this opportunity. We also thank laboratory staffs of Debre Berhan public heath institution for their help during data collection process.

\section{References}

[1] Msuya SE, Uriyo J, Hussain A, et al. Prevalence of sexually transmitted infections among pregnant women with known HIV status in northern Tanzania. Reprod Health. 2009;6:4.

[2] Minichiello V, Rahman S, Hussain R. Epidemiology of sexually transmitted infections in global indigenous populations: data availability and gaps. Int J STD AIDS. 2013;24(10):759-768.

[3] The World Bank. Sexually transmitted infections in developing countries: current concepts and strategies on improving STI prevention, treatment, and control [webpage on the Internet] Washington, DC: The World Bank; 2008.

[4] Jolly DH, Mueller MP, Chen M, et al. Concurrency and other sexual risk behaviors among black young adults in a southeastern city. AIDS Educ Prev 2016; 28(1):59-76.

[5] Dolwick Grieb SM, Davey-Rothwell M, Latkin CA. Concurrent sexual partnerships among urban African American high risk women with main sex partners. AIDS Behav 2012; 16(2):323-333. DOI: 10. 1007/s10461-01199546.

[6] Hogben M, Leichliter JS. Social determinants and sexually transmitted disease disparities. Sex Transm Dis 2008; 35(12):S13- S18.

[7] Fatima N, Malik A, Khan PA, Ali S, Khan HM. Sero prevalence of syphilis infection among patients attending Antenatal Care \& Sexually transmitted disease (STD) clinics : observations from a tertiary care hospital of northern India. 2014: 2 (1):6-9.

[8] World Health Organization Guidelines for the Treatment of Treponema Pallidum (Syphilis). 2016.

[9] Chiduo M, Theilgaard ZP, Bakari V, et al. Prevalence of sexually transmitted infections among women attending antenatal clinics in Tanga, northeastern Tanzania. Int J STD AIDS. 2012;23(5):325-329.

[10] Frickmann H, Schwarz NG, Girmann M, et al. Serological survey of HIV and syphilis in pregnant women in Madagascar. Trop Med Int Health. 2013;18(1):35-39.

[11] Ramos JM, Toro C, Reyes F, Amor A, Gutiérrez F. Seroprevalence of HIV-1, HBV, HTLV-1 and Treponema pallidum among pregnant women in a rural hospital in Southern Ethiopia. J Clin Virol. 2011;51(1):83-85. 
[12] Federal Democratic Republic of Ethiopia Minestry of health: Report on the 2014 Round Antenatal Care based Sentinel HIV Surveillance in Ethiopia. 2015;(July).

[13] Magnitude of HIV and syphilis seroprevalence among pregnant women in Gondar, Northwest Ethiopia: a crosssectional study. 2015;75-82.

[14] Endris M, Deressa T, Belyhun Y, Moges F. Seroprevalence of syphilis and human immunodeficiency virus infections among pregnant women who attend the University of Gondar teaching hospital, Northwest Ethiopia : a cross sectional study. 2015;1-7.

[15] African E, Journal M. Prevalence of Syphilis in Pregnancy in Addis Ababa. 2000; 77 (4):212-6.

[16] Federal Democratic Republic of Ethiopia Minestry of health: Report on the 2014 Round Antenatal Care based Sentinel HIV Surveillance in Ethiopia. 2015;(July).

[17] Chegen A. Assessment of magnitude of HIV and syphilis and associated factors among pregnant women attending antenatal care in Wolmera district, Addis Ababa university, college of health sciences:2017(Un published).
[18] Mulu A, Kassu A, Tessema B, Yismaw G, Tiruneh M. Seroprevalence of Syphilis and HIV-1 during Pregnancy in a Teaching Hospital in Northwest Ethiopia. 2007;193-5.

[19] Federal Democratic Republic of Ethiopia Minestry of health: Report on the 2009 Round Antenatal Care Sentinel HIV Surveillance in Ethiopia. 2011.

[20] Dionne-odom J, Mbah R, Rembert NJ, Tancho S, Halle-ekane GE, Enah C, et al. Hepatitis, HIV, and Syphilis Seroprevalence in Pregnant Women and Blood Donors in Cameroon. 2016;2016.

[21] World Health Organization (WHO): HIV in the African region, 2013; 13-14.

[22] EPHI. Report on the 2014 Round Antenatal Care based Sentinel HIV Surveillance in Ethiopia. Addis Ababa: The Ethiopia public health institution 2015.

[23] Kwiek JJ, Mwapasa V, Alker AP, Muula AS. Sociodemographic characteristics associated with HIV and syphilis seroreactivity among pregnant women in Blantyre, Malawi, 2000-2004. Malawi Medical Journal. 2004 2008; 20(3):80-5. 